\title{
The Electrorheological Properties of Polypyrrole Suspensions
}

\author{
Shuizhu Wu, Fang ZeNG, ${ }^{*}$ and Jiarui SHEN \\ Department of Polymer Science \& Engineering, South China University \\ of Technology Guangzhou 510641, People's Republic of China \\ * The Research Institute of Materials Science, South China University \\ of Technology Guangzhou 510641, People's Republic of China
}

(Received July 7, 1997)

\begin{abstract}
In this article, the electrorheological (ER) suspensions containing polypyrrole particles in silicon oil are prepared. These suspensions' ER properties, in particular their conductive property and the influencing factors, such as particle conductivity, field strength, concentration and shear rate, are investigated experimentally. The results show that the suspension can display significant ER effects only when the particle conductivity is within a certain range, and the suspension's ER effect increases with particle concentration and field strength, but decreases with the increasing shear rate. The suspension's current density increases with particle concentration, particle conductivity and field strength, while decreases with shear rate.
\end{abstract} KEY WORDS Polypyrrole / Conductive Property / Suspension / Electrorheological Effect /

The electrorheological (ER) effect is noted for a rapid and reversible change in suspension fluidity upon application of an electric field. Since its discovery, ${ }^{1,2}$ it has been recognized to have great potential engineering applications, thus attracts lasting interest in the research of electrorheology. However, there are still problems to be solved before ER systems find extensive commercial application. It has been long observed that wet particulates are most ER active. But these moist fluids are limited to a narrow temperature range and show undesirable levels of conductance arising from mobile ions. ${ }^{3,4}$ Solution of these problems and the development of better ER fluids depend on improving our understanding of how the phenomenon depends upon the properties of the materials which make up ER fluids. Recent development of anhydrous suspensions based on conducting materials seems to have overcome some of these problems. ${ }^{4,5}$

The basic idea to design the water-free ER fluids is to control the ability of polarization, i.e., to find electronic semiconductors with appropriate conductivity as particles which provide large interfacial polarization when surrounded by an insulating surface in anhydrous suspensions. Along this line of thinking we prepared the ER suspensions containing polypyrrole particles with different conductivity, and studied the effects of particle conductivity on ER effect.

The conductance of ER fluid is believed to be an important parameter in ER effect especially when it comes to practical applications. Large increase in the conductance of the suspension would result in excessive power demands with possible serious implications in terms of power supply and energy dissipation in the ER device, it could even cause dielectric breakdown. ${ }^{6,7}$ For this reason, low conductance is an important goal for future ER fluids. Therefore, in this article, the conductive property of the ER suspension and its influencing factors are investigated as well, which might give some information on future applications of ER suspensions.

\section{EXPERIMENTAL}

\section{Synthesis and Suspension Preparation}

Under $20^{\circ} \mathrm{C}$, a certain amount of $\mathrm{FeCl}_{3}$ was added to the distilled water $(600 \mathrm{ml})$ in a round bottom flask which is fitted with a reflux condenser. Pyrrole (Fluka) $(0.07 \mathrm{~mol})$ was then added in a single portion to the stirred $\mathrm{FeCl}_{3}$ solution. Upon the addition of pyrrole monomer, a reaction exotherm was observed (temperature increased up to about $35^{\circ} \mathrm{C}$ ) and the solution instantly acquired a black color as the precipitate of polypyrrole formed. After a reaction time of $24 \mathrm{~h}$ at $20^{\circ} \mathrm{C}$, the mixture was filtered, and the precipitate was washed with large amounts of distilled water, followed by a wash with dilute $\mathrm{HCl}$ solution. The product was then rinsed with distilled water again until the washings tested neutral with $\mathrm{pH}$ paper. Finally, the product was washed with ethanol, and the black powder was dried in vacuum at $80^{\circ} \mathrm{C}$.

After drying, a certain amount of the polypyrrole samples (the density of polypyrrole powder is about 1.4) were dispersed in a given amount of silicon oil, then the mixture was ball milled until microscopic examination indicated a mean particle size $<10 \mu \mathrm{m}$ and the absence of particles $>20 \mu \mathrm{m}$. Particles were irregular in shape but without any tendency to anisometry. The silicone oil used is a colorless oil with the following physical properties: density $0.97 \mathrm{~g} \mathrm{~cm}^{-3}$, viscosity $100 \mathrm{mPas}$ at $20^{\circ} \mathrm{C}$, dielectric constant 2.8 , and boiling temperature $300^{\circ} \mathrm{C}$. There was little tendency for these dispersions to separate in the short term, and such dispersions that had separated after lengthy standing readily redispersed on agitation.

\section{Methods of Measurement}

For conductivity measurements, pellets of each sample of dry polypyrrole were pressed at $1.8 \times 10^{7} \mathrm{~Pa}$, coated top and bottom with gold and placed between two electrodes, $0.01 \mathrm{~V}$ was applied, and the current was measured. The conductivity was calculated using surface area and the thickness of each pellet.

For ER measurement, a concentric cylinder rheometer was used. To apply large electric field strength across the concentric cylinders, each cylinder was insulated 
from the rest of the rheometer. The inner cylinder has an outer diameter of $14.6 \mathrm{~mm}$ and height of $30 \mathrm{~mm}$. The outer cylinder has an inner diameter of $20 \mathrm{~mm}$ and height of $35 \mathrm{~mm}$. The annual gap is $2.7 \mathrm{~mm}$. The electric field strength was applied to the gap by grounding the outer cylinder and connecting the inner cylinder to a highvoltage source. The DC voltages in the range of 100 $2500 \mathrm{~V}$ were used. The voltage was monitored using a multimeter attached to a one-tenth voltage readout connection on the power supply. The current was monitored using a multimeter attached in series to the ground wire of the circuit. The current density was calculated through dividing the measured current by the surface area of the electrode. All measurements were carried out at room temperature.

\section{RESULTS AND DISCUSSION}

\section{Effects of Particle Conductivity}

During the synthesis of pyrrole, ferric chloride functions both as polymerization initiator and as dopant in converting pyrrole into electrically conducting polymer. In order to obtain polypyrrole samples with different conductivity, we change the reactant ratio, i.e., $\mathrm{FeCl}_{3} /$ pyrrole mole ratio, during the polymerization process. The conductivity of the $\mathrm{FeCl}_{3}$-doped polypyrroles as a function of the reactant ratio is shown in Table I.

The ER effects of the suspensions containing polypyrrole particles with different conductivity are shown in Figure 1. It can be seen that, as particle conductivity increases, the suspension displays much more significant ER effect (greater enhancement of shear stress or viscosity). And the suspension containing polypyrrole particles with a conductivity of about $10^{-10} \mathrm{~S} \mathrm{~cm}^{-1}$ barely displays any ER effect. In this experiment, we also found that for suspensions containing polypyrrole particles with a conductivity of over $10^{-6} \mathrm{~S} \mathrm{~cm}^{-1}(1: 1$ reactant ratio), the suspensions' current density is very high. As a consequence of instrumental limitation, no ER data could be collected on these samples. The suspension based on particles with a conductivity of about $10^{-10} \mathrm{~S} \mathrm{~cm}^{-1}$ is rather ineffective, this probably is because of the low particle conductivity and its consequent slow and poor level polarization. Only within the particle conductivity range of between $10^{-6} \mathrm{~S} \mathrm{~cm}^{-1}$ and $10^{-10} \mathrm{~S} \mathrm{~cm}^{-1}$, could the suspension exhibit significant ER effect, and its ER effect increases with the particle conductivity within this range. These experimental results indicate that the particle conductivity is an important factor affecting the suspension's ER effect.

The current density in the suspensions containing different polypyrrole samples is shown in Figure 2. Figure 2 indicates that the current density increases with the increasing particle conductivity, suggesting that the particles themselves carry current. This experimental result is similar with that of the polyaniline ER system by Gow and Zukoski. ${ }^{8}$ According to the fibrillation theory, ${ }^{1,2,9}$ under the applied electric field, the dispersed particles form chains or fibrils, which bridge the gap between the electrodes. This suspension structure leads to the field enhancement of shear stress or viscosity (ER effect). These structures bridging the electrodes
Table I. Reactant ratio effects on polypyrrole samples' conductivity

\begin{tabular}{cc}
\hline $\mathrm{FeCl}_{3} /$ pyrrole mole ratio & Conductivity/S cm \\
\hline $1: 10$ & $2.0 \times 10^{-10}$ \\
$1: 8$ & $3.0 \times 10^{-9}$ \\
$1: 6$ & $3.2 \times 10^{-8}$ \\
$1: 5$ & $9.0 \times 10^{-8}$ \\
$1: 3$ & $2.1 \times 10^{-7}$ \\
$1: 2$ & $5.1 \times 10^{-7}$ \\
$1: 1$ & $5.2 \times 10^{-6}$ \\
$2: 1$ & $6.1 \times 10^{-5}$ \\
$3: 1$ & $1.7 \times 10^{-4}$ \\
$4: 1$ & $3.1 \times 10^{-4}$ \\
$6: 1$ & $3.6 \times 10^{-4}$ \\
\hline
\end{tabular}

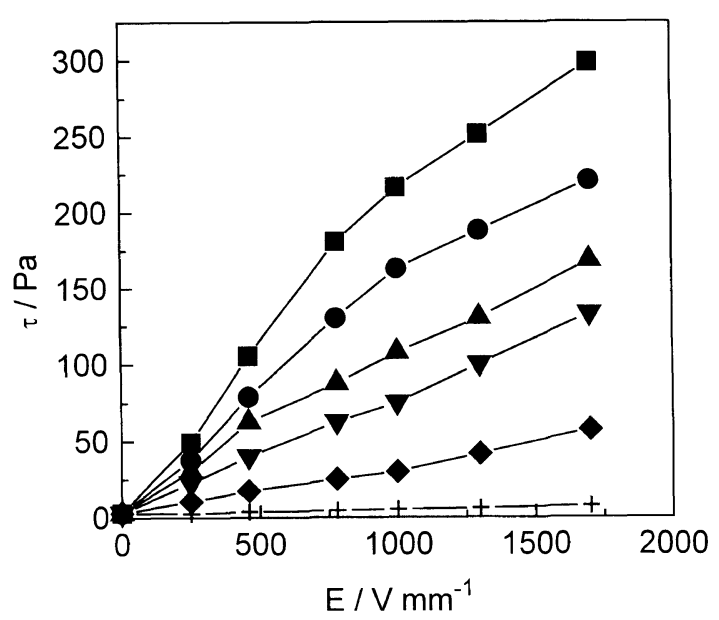

Figure 1. The suspension's shear stress versus field strength curve. $\left(C=15 \mathrm{wt} \% ; \gamma=2.5 \mathrm{~s}^{-1}\right.$. $)-(\square)-, 5.1 \times 10^{-7} \mathrm{~S} \mathrm{~cm}^{-1} ;-(\mathbf{O})-, 2.1 \times$ $10^{-7} \mathrm{~S} \mathrm{~cm}^{-1} ;-(\Delta)-, 9.0 \times 10^{-8} \mathrm{~S} \mathrm{~cm}^{-1} ;-(\boldsymbol{\nabla})-, 3.2 \times 10^{-8} \mathrm{~S} \mathrm{~cm}^{-1}$; $-(\diamond)-, 3.0 \times 10^{-9} \mathrm{~S} \mathrm{~cm}^{-1} ;-(+)-, 2.0 \times 10^{-10} \mathrm{~S} \mathrm{~cm}^{-1}$.

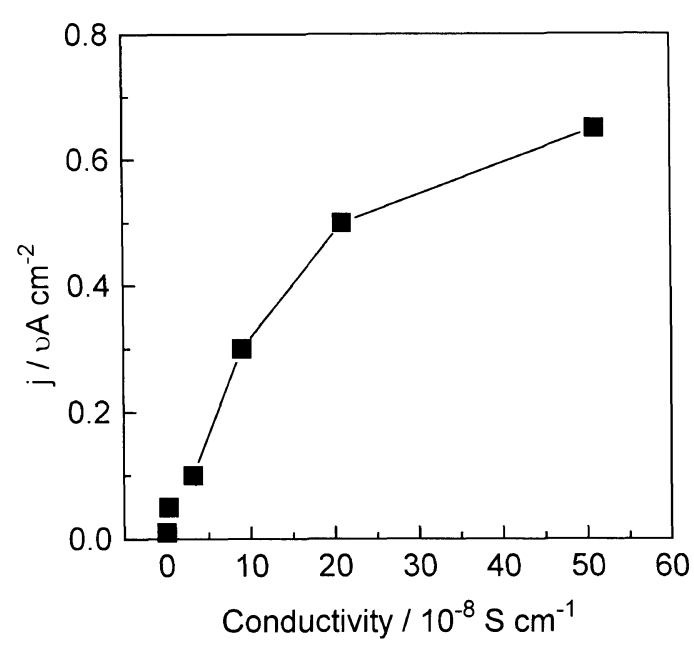

Figure 2. The suspension current density versus particle conductivity curve. $\left(C=15 \mathrm{wt} \% ; E=2000 \mathrm{~V} \mathrm{~mm}^{-1} ; \dot{\gamma}=0 \mathrm{~s}^{-1}\right.$.)

would provide conducting pathways. And since polypyrrole is an electronic semiconductor, electron transport between particles is possible at the high fields and concentrations of particulates used. Charge transport may involve tunneling or hopping between closely spaced particles. it is also possible that the particles themselves constitute the carriers by capturing charge from one electrode and transporting it either via charge transfer between particles or directly by moving to the opposite 


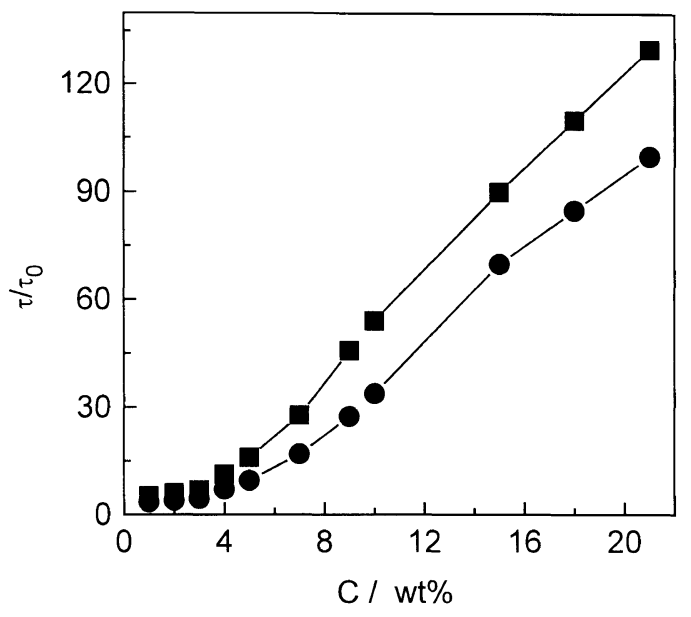

Figure 3. The relative shear stress as a function of particle concentration. $\left(\dot{\gamma}=2.5 \mathrm{~s}^{-1}\right.$.) -(口)-, $1500 \mathrm{~V} \mathrm{~mm}^{-1} ;-(\bigcirc)-, 1000 \mathrm{~V} \mathrm{~mm}^{-1}$.

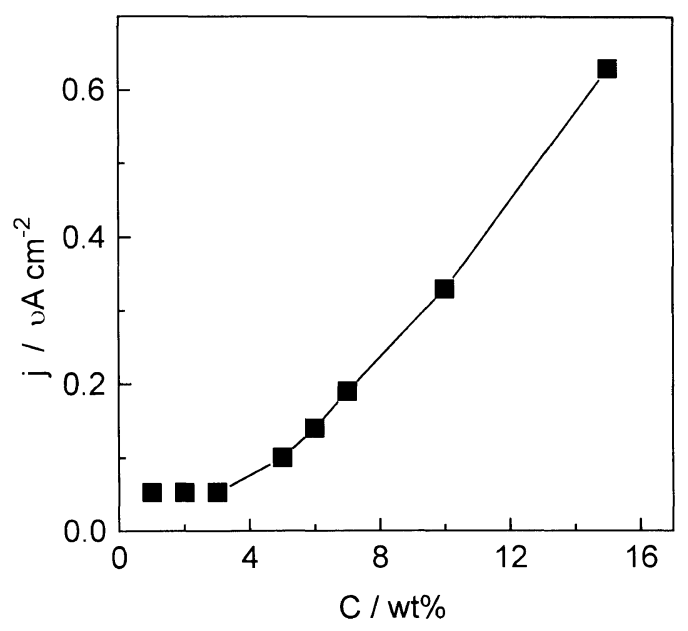

Figure 4. The suspension's current density versus particle concentration curve. $\left(E=2500 \mathrm{~V} \mathrm{~mm}^{-1}, \dot{\gamma}=0 \mathrm{~s}^{-1}\right.$.)

electrode. As shown above, the suspension's ER effect increases with the increasing particle conductivity. Therefore, for dispersions based on more highly conductive materials, the level of conductance through the dispersion increases.

\section{Effects of Particle Concentration}

Figure 3 shows the relative shear stress of the suspension at different concentrations. The relative shear stress means the ratio of the suspension's shear stress to zero-field shear stress under the same shear rate. And the current density as a function of particle concentration is shown in Figure 4.

It seems that there exists a critical concentration, under which the suspension barely displays any ER effect. Over the critical concentration, the suspension's ER effect increases with the increasing concentration. The formation of particle chains or strands is a percolation process. ${ }^{10}$ Only when there are enough particles in the dispersion, should the particles strands or chains span the electrode gap. The shear field distorts the suspension structure (particle strands or chains), and the energy expended in this deformation results in the observed enhancement of shear stress or viscosity. This is why there is a critical concentration. Above the critical

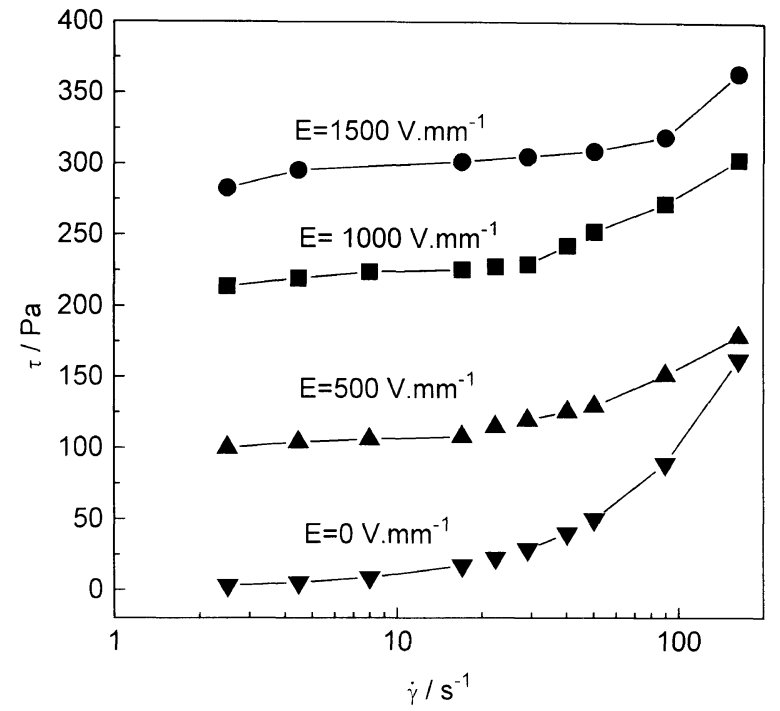

Figure 5. The suspension's shear stress as a function of shear rate at different field strengths. $(C=15 \mathrm{wt} \%$; particle conductivity $=5.1 \times$ $10^{-7} \mathrm{~S} \mathrm{~cm}^{-1}$.)

concentration, the amount of particle strands bridging the electrodes increases with the increasing amount of particles (concentration), which in turn provides more conducting pathways. Hence, the current passing through the suspension increases with concentration. Under the critical concentration, the dispersed particles could only form short chains. There are less possible conducting pathways in the suspension, therefore, small current passes the dispersion.

\section{Effects of Field Strength}

The suspension's shear stress as a function of shear rate at different field strengths is shown in Figure 5. It can be seen that, at zero-field strength, the suspension's shear stress is much lower than that of the suspensions under the applied electric field strength, and at high shear rates, the suspension's viscosity almost approaches a constant value; While under the applied field strength, the suspension's shear stress increases significantly, and as the shear rate decreases, the shear stress reaches a plateau. The shear rate at which the plateau begins and the magnitude of the plateau stress depend on the magnitude of the electric field strength. At the small shear rate, the stress is independent of shear rate. This indicates that in this small shear rate regime, the stress transfer is purely electrostatic $\left(\tau=\tau_{0}(E)\right.$ when $\dot{\gamma}$ is small).

At very large shear rates, the suspension viscosity nearly approaches a constant value. Under this condition, viscous forces are much larger than polarization forces and the fibrous structures are greatly degraded. As a result, the stress is thus transferred by mainly viscous means $(\tau=\eta \dot{\gamma}$, when shear rate is very large).

At the intermediate shear rates, however, hydrodynamic and electrostatic polarization contributions to the shear stress are of comparable magnitudes. So, there might be a synergistic interaction between hydrodynamic and electromechanical stress transfer due to the indirect influence one mode will have on the other mode through alterations in the suspension structure $(\tau=\tau(\dot{\gamma}, E)$ when shear rate is intermediate). A transition from the small 


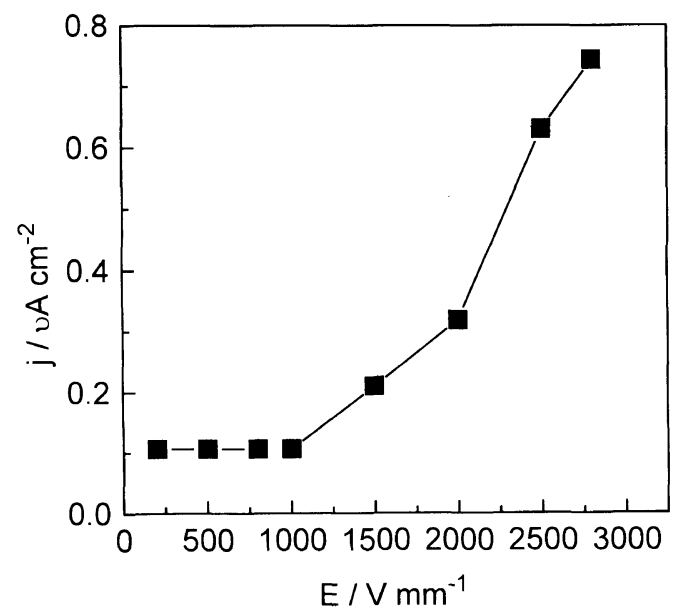

Figure 6. Suspension's current density as a function of field strength. $\left(C=15 \mathrm{wt}^{0} \% ; \dot{\gamma}=0 \mathrm{~s}^{-1}\right.$.)

shear rate region to the large shear rate regime corresponds to the transition from flow patterns of the system with a non-destroyed structure to that with a greatly damaged structure.

The suspension's current density under different field strengths is shown in Figure 6. Within the lower field strength range, the current passing through the suspension is relatively low. However, at higher electric field strength, the current density increases rapidly with the increasing field strength. This phenomenon is because, under lower field strength, there are not many particle strands or chains which bridge the electrodes. So, there are not many conducting pathways. While under higher field strength, the polarization forces between the dispersed particles are much stronger, thus leads to more particle chains or strands spanning the gap between the electrodes, which results in more possible conducting pathways. Furthermore, under higher field strength, the space charge current could occur due to the electrons discharged into the fluid from the electrode. The space charge current is non-ohmic, and proportional to the square of the field strength. ${ }^{11}$ Under higher field strength, the space charge current could contribute to the current density of the suspension.

\section{Effects of Shear Rate}

The current density of the suspension as a function of shear rate is shown Figure 7. It can be noted that, under lower shear rate range, the current density barely changes and remains relatively high; while at higher shear rate range, the suspension's current density decreases rapidly with the increasing shear rate.

The polarization forces between the suspended particles induced by the applied electric field lead to the formation of particle strands or chains, which in turn results in the increased shear stress or viscosity (ER effect). In the presence of a shear field simultaneously, the dispersed particles are also acted on by the viscous forces, which will rupture the particle chains or strands. The viscous forces scale as $6 \pi \eta a^{2} \dot{\gamma}$, where $\eta$ is the viscosity

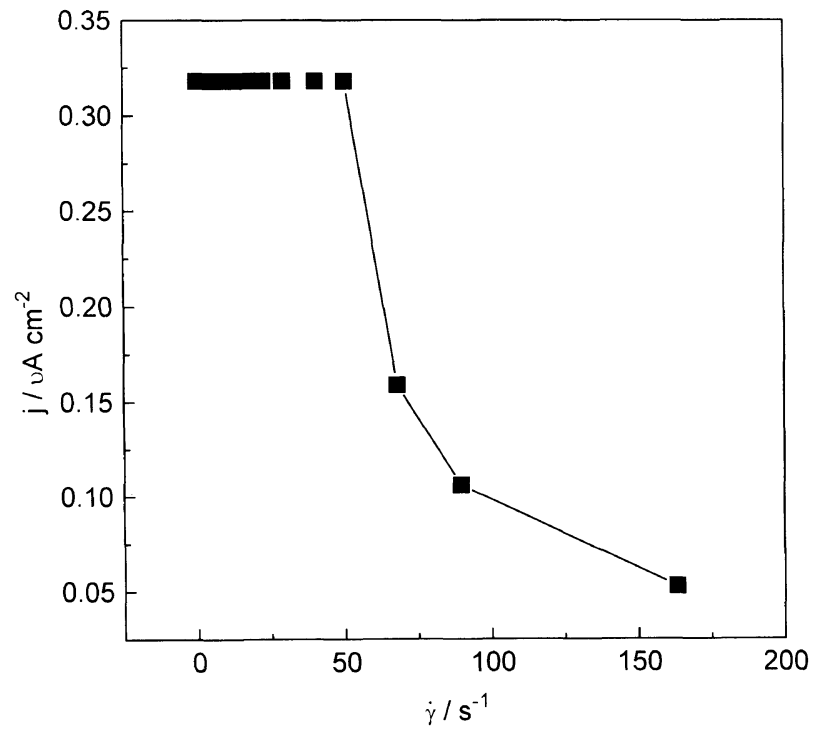

Figure 7. The suspension's current density as a function of shear rate $\left(C=15 \mathrm{wt}^{\circ} ; E=2000 \mathrm{~V} \mathrm{~mm}^{-1}\right.$.)

of the dispersing fluid, $\dot{\gamma}$ is the shear rate, and $a$ is the radius of the particle. ${ }^{9}$ It can be seen that, as the shear rate is increased, the viscous forces acted upon the particles increase, so that the tendency to breakdown the structural skeleton of the suspension is enhanced.

At lower shear rate, the polarization forces remains dominant, little damage is done to the suspension structure (particle strands or chains), therefore, the suspension could display significant ER effect and the current density remains relatively high; While with the enhancing shear rate, the viscous forces gradually take hold, thus causes more ruptured particle strands or chains, so the amount of the possible conducting pathways decreases as well, therefore, the current density of the suspension decreases.

Acknowledgment. The authors gratefully acknowledge the support of Guangdong Natural Science Foundation and Guangdong High Education Bureau of China.

\section{REFERENCES}

1. W. M. Winslow, U. S. Patent 2417850 (1947).

2. W. M. Winslow, J. Appl. Phys., 20, 1137 (1949).

3. D. Scott and J. Yamaguchi, Automotive Engineering, 91, 61 (1983).

4. H. Block and J. P. Kelly, J. Phys. D, 21, 1661 (1988).

5. H. Block and J. P. Kelly, U. S. Patent 4687589 (1987).

6. H. Block, J. P. Kelly, A. Qin, and T. Watson, Langmuir, 6, 6 (1990).

7. D. L. Klass and T. W. Martinek, J. Appl. Phys., 38, 75 (1967).

8. C. J. Gow and C. F. Zukoski, J. Colloid Interface Sci., 136, 175 (1990).

9. A. P. Gast and C. F. Zukoski, Adv. Colloid Interface Sci., 30, 153 (1989).

10. D. J. Klingenberg and C. F. Zukoski, J. Chem. Phys., 91, 7888 (1989).

11. R. Coelho, "Physics of Dielectrics for the Engineer," Elsevier, London, 1979. 